Article

\title{
Low-Temperature Sol-Gel Synthesis of Nitrogen-Doped Anatase/Brookite Biphasic Nanoparticles with High Surface Area and Visible-Light Performance
}

\author{
Liang Jiang, Yizhou Li, Haiyan Yang, Yepeng Yang, Jun Liu, Zhiying Yan, Xiang Long, Jiao He and \\ Jiaqiang Wang * \\ National Center for International Research on Photoelectric and Energy Materials (MOST), Yunnan Provincial \\ Collaborative Innovation Center of Green Chemistry for Lignite Energy, Yunnan Province Engineering Research \\ Center of Photocatalytic Treatment of Industrial Wastewater, The Universities' Center for Photocatalytic \\ Treatment of Pollutants in Yunnan Province, School of Energy, School of Chemical Sciences \& Technology, \\ Yunnan University, Kunming 650091, China; liangjiang_ynu@163.com (L.J.); zh111111ou@sina.com (Y.L.); \\ ashoulu@sina.com (H.Y.); mondaysunday1234@163.com (Y.Y.); 18468068607@163.com (J.L.); \\ zhyyan@ynu.edu.cn (Z.Y.); lxbl1991@163.com (X.L.); hejiao@ynu.edu.cn (J.H.) \\ * Correspondence: jqwang@ynu.edu.cn; Tel.: +86-871-6503-1567
}

Received: 21 October 2017; Accepted: 30 November 2017; Published: 4 December 2017

\begin{abstract}
Nitrogen doping in combination with the brookite phase or a mixture of $\mathrm{TiO}_{2}$ polymorphs nanomaterials can enhance photocatalytic activity under visible light. Generally, nitrogendopedanatase/brookite mixed phases $\mathrm{TiO}_{2}$ nanoparticles obtained by hydrothermal or solvothermal method need to be at high temperature and with long time heating treatment. Furthermore, the surface areas of them are low $\left(<125 \mathrm{~m}^{2} / \mathrm{g}\right)$. There is hardly a report on the simple and direct preparation of $\mathrm{N}$-doped anatase/brookite mixed phase $\mathrm{TiO}_{2}$ nanostructures using sol-gel method at low heating temperature. In this paper, the nitrogen-doped anatase/brookite biphasic nanoparticles with large surface area $\left(240 \mathrm{~m}^{2} / \mathrm{g}\right)$ were successfully prepared using sol-gel method at low temperature $\left(165^{\circ} \mathrm{C}\right)$, and with short heating time $(4 \mathrm{~h})$ under autogenous pressure. The obtained sample without subsequent annealing at elevated temperatures showed enhanced photocatalytic efficiency for the degradation of methyl orange (MO) with 4.2-, 9.6-, and 7.5-fold visible light activities compared to P25 and the amorphous samples heated in muffle furnace with air or in tube furnace with a flow of nitrogen at $165^{\circ} \mathrm{C}$, respectively. This result was attributed to the synergistic effects of nitrogen doping, mixed crystalline phases, and high surface area.
\end{abstract}

Keywords: anatase/brookite biphasic; nitrogen-doping; sol-gel method; visible light photocatalysis; degradation of dyes

\section{Introduction}

Heterogeneous photocatalytic processes involving $\mathrm{TiO}_{2}$ semiconductor particles have been shown to be a promising process for the treatment of dye effluents [1]. However, large band gap energy $(3.2 \mathrm{eV})$ for anatase $\mathrm{TiO}_{2}$ limits its practical application for natural solar applications [2]. To develop more light-efficient catalysts, there is an urgent need to develop photocatalytic systems which are able to operate effectively under visible light irradiation. A number of systems have been reported to improve the visible-light activity of $\mathrm{TiO}_{2}$. Meanwhile, selecting the reasonable substrate and activity test are helpful to systematically and comprehensively assess the photocatalytic efficiency of the catalysts [3]. Nitrogen-doped (N-doped) $\mathrm{TiO}_{2}$ is one of the most typical examples of the visible-light photocatalysts, which is due to nitrogen doping can decrease the band gap energy and enhance the 
photoactivity of $\mathrm{TiO}_{2}$ in the visible spectral range [4,5]. However, the low reactivity and quantum efficiency of $\mathrm{N}$-doped $\mathrm{TiO}_{2}$ limit its practical application [6,7].

On the other hand, $\mathrm{TiO}_{2}$ exists in three main polymorphs, which are anatase, rutile, and brookite [8,9]. Phase mixing is well recognized as the most promising strategy for quantum efficiency improvement, which can be due to the enhanced charge carrier separation [6,10-14]. Particularly, it has been proven that the mixed phase of anatase/rutile $\mathrm{TiO}_{2}$ has synergistic effects and higher photocatalytic activity as compared to pure phase of either in anatase or rutile $[15,16]$. In contrast to anatase/rutile biphasic nanoparticles which have been intensively studied, the photocatalytic study of brookite and its phase mixing is quite limited, though it has been reported that anatase/brookite mixed-phase $\mathrm{TiO}_{2}$ has higher activity in visible light than P25 [8]. The reason may be mainly due to the difficulties in synthesis [17]. For example, anatase-brookite composite nanocrystals were synthesized by a sonochemical sol-gel method at very high heating temperature $\left(500{ }^{\circ} \mathrm{C}\right)[12,18]$. Highly crystalline phase-pure brookite and anatase/brookite mixed-phase $\mathrm{TiO}_{2}$ nanostructures were synthesized via a simple hydrothermal method with titanium sulphide as the precursors in sodium hydroxide solutions [19]. Interestingly, anatase-brookite heterojunction $\mathrm{TiO}_{2}$ photocatalysts were purposefully tailored by introducing different glycine concentrations through hydrothermal treatment at $200{ }^{\circ} \mathrm{C}$ for $20 \mathrm{~h}[20]$.

It is expected that a strategy coupling a binary structure with nitrogen doping could bring enhanced photocatalytic properties of $\mathrm{TiO}_{2}$. Recently, $\mathrm{N}$-doped anatase/rutile $\mathrm{TiO}_{2}$ nanoparticles have been designed and synthesized [19,21]. Anatase/brookite mixed-phase nitrogen-doped $\mathrm{TiO}_{2}$ nanoparticles were also synthesized by a facile solvothermal route [22]. Interestingly, nitrogen plasma treatment was employed to prepare $\mathrm{N}$-doped nanoporous $\mathrm{TiO}_{2}$ with large surface area and high-crystalline anatase/brookite phase [23].

Generally, a semiconductor catalyst with large specific surface area is beneficial for efficient photocatalysis, while in most synthetic processes, $\mathrm{TiO}_{2}$ with the brookite phase or a mixture of $\mathrm{TiO}_{2}$ polymorphs obtained hydrothermally at high temperature and with long time heat treatment have low surface area $[14,19,23,24]$. Hence, it is challenging to synthesize $\mathrm{N}$-doped anatase/brookite $\mathrm{TiO}_{2}$ photocatalyst with large surface area and enhanced visible light activity at low temperature via simple and direct synthetic method.

Sol-gel is one of the most prominent methods used to prepare mixed phases of anatase/rutile $\mathrm{TiO}_{2}$ nanoparticles due to its simplicity and low equipment requirements. However, there are few reports on the simple and direct preparation of $\mathrm{N}$-doped anatase/brookite mixed phase $\mathrm{TiO}_{2}$ nanostructures using sol-gel method at low heating temperature $[15,16]$. The goal of the present work is to synthesize anatase/brookite biphasic $\mathrm{TiO}_{2}$ nanoparticles by direct introduction of nitrogen in $\mathrm{TiO}_{2}$ lattice crystal during the sol-gel preparation at low temperature. In this work, the degradation of methyl orange (MO) in aqueous solution under visible light irradiations was selected to test the enhanced photocatalytic efficiency. It has been reported that amorphous $\mathrm{TiO}_{2}$ or a mixture composed of crystalline and amorphous $\mathrm{TiO}_{2}$ has high activity for the photocatalytic degradation of pollutants $[25,26]$. However, synthesized nitrogen-doped anatase/brookite biphasic nanoparticles of this work exhibited much higher photocatalytic efficiency than the prepared amorphous samples.

\section{Results and Discussion}

\subsection{Syntheses and Characterizations}

The synthesis process of this work was modified from a typical sol-gel method by using $\mathrm{HNO}_{3}$-catalyzed hydrolysis step of titanium tetraisopropoxide (TTIP) to reduce the hydrolysis rates [27]. Generally, heating is required to prepare crystalline $\mathrm{TiO}_{2}$. If low heating temperature in the range of $180-200{ }^{\circ} \mathrm{C}$ was employed in hydrothermal or solvothermal method, longer time (3-48 h) would be needed. Nevertheless, the obtained $\mathrm{TiO}_{2}$ samples still have low surface area $\left(<125 \mathrm{~m}^{2} / \mathrm{g}\right)[18,21,23]$. Moreover, a supercritical drying process was often used in the conventional 
sol-gel method [28]. By contrast, herein the aged gels were heated under nitrogen atmosphere with a much lower final autogenous pressure (about $350 \mathrm{psi}$ ), heating temperature $\left(165^{\circ} \mathrm{C}\right)$, and shorter heating time $(4 \mathrm{~h})$.

The crystal structures of sample NA-185 and NA-165 with anatase and brookite phases were identified by X-ray diffraction (XRD), as shown in Figure 1. The diffraction peaks of $2 \theta$ values at $25.3^{\circ}, 37.8^{\circ}, 48.1^{\circ}, 54.9^{\circ}, 62.8^{\circ}, 69.8^{\circ}, 75.4^{\circ}, 82.8^{\circ}$ are assigned to the (101), (004), (200), (204), (220), (215), and (224) planes of anatase $\mathrm{TiO}_{2}$ (JCPDS 21-1272). Due to the overlapping of the planes of brookite (120), brookite (111), and anatase (101), the existence of the brookite phase was determined by the brookite (121) plane at $30.8^{\circ}$ (JCPDS 29-1360). Both NA-145 and HA-165 are phase-pure anatase. From the XRD peak intensities [29], the brookite phase contents of sample NA-185 and NA-165 were calculated to be $\sim 10 \%$ and $\sim 6 \%$, respectively. The crystal size was calculated by Scherrer equation (Table 1).

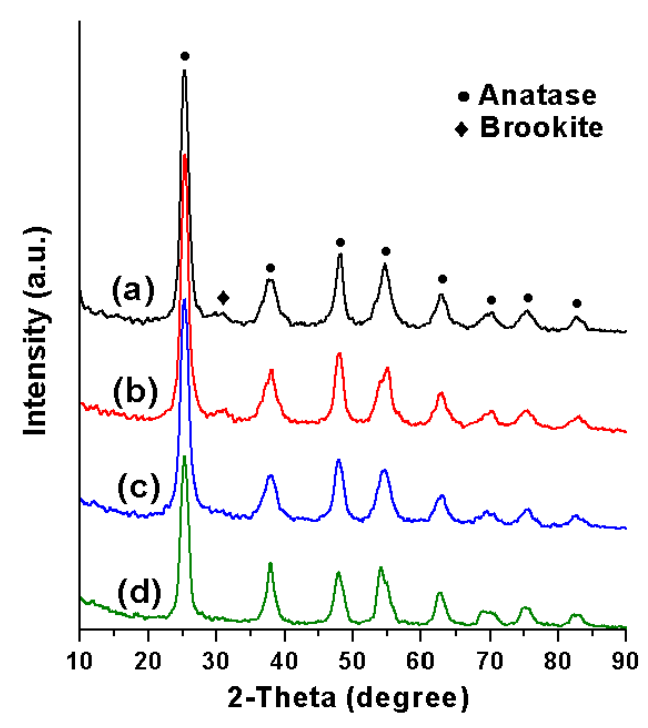

Figure 1. X-ray diffraction (XRD) patterns of as-prepared samples: (a) NA-185, (b) NA-165, (c) NA-145, (d) HA-165.

Table 1. The characteristics and the apparent first-order rate constant $\mathrm{K}\left(\mathrm{min}^{-1}\right)$ of samples.

\begin{tabular}{|c|c|c|c|c|c|c|}
\hline \multirow{2}{*}{ Samples } & \multicolumn{2}{|c|}{ Anatase } & \multicolumn{2}{|c|}{ Brookite } & \multirow[t]{2}{*}{$\mathrm{S}_{\text {BET }}\left(\mathrm{m}^{2} / \mathrm{g}\right)$} & \multirow{2}{*}{$K\left(\min ^{-1}\right)$} \\
\hline & Crystal Size $^{\text {a }}(\mathrm{nm})$ & Content $^{b}(\%)$ & Crystal Size $^{\text {a }}(\mathrm{nm})$ & Content $^{b}(\%)$ & & \\
\hline NA-185 & 6.7 & 90 & 7.3 & 10 & 239 & 0.023 \\
\hline NA-165 & 7.5 & 94 & 7.2 & 6 & 240 & 0.021 \\
\hline NA-145 & 6.5 & 100 & - & - & 249 & 0.015 \\
\hline HA-165 & 7.9 & 100 & - & - & 216 & 0.006 \\
\hline TF-165 & - & - & - & - & 443 & 0.002 \\
\hline MF-165 & - & - & - & - & 407 & 0.003 \\
\hline P25 & - & - & - & - & 50 & 0.005 \\
\hline
\end{tabular}

${ }^{\mathbf{a}}$ Determined by the Scherrer equation; ${ }^{\mathbf{b}}$ Calculated using the formula in reference [29].

The morphology and particle size of the samples revealed by Scanning electron microscopy (SEM) and Transmission electron microscopy (TEM) analysis (Figures S1 and S2). NA-185, NA-165, NA-145, and HA-165 all show aggregates consisting of small spheroidal nanoparticles with average size of approximately 6-8 $\mathrm{nm}$, which was in agreement with the results calculated by Scherrer equation. To further confirm the existence of anatase and brookite phases, high-resolution TEM (HRTEM) analysis of NA-165 was performed. As shown in Figure 2a,b, the lattice fringes of $0.35 \mathrm{~nm}$ and $0.29 \mathrm{~nm}$ match the anatase (101) and brookite (121) plane, respectively. The results are in agreement with the XRD observations. 


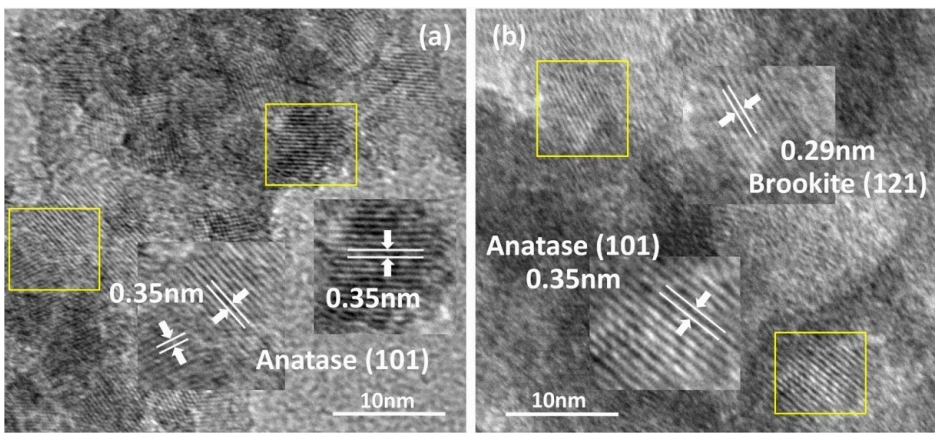

Figure 2. High-resolution TEM (HRTEM) images of NA-165. The lattice fringes of $0.35 \mathrm{~nm}$ and $0.29 \mathrm{~nm}$ match (a) the anatase (101) and (b) brookite (121) plane.

The X-ray photoelectron spectroscopy (XPS) measurements reveal the surface compositions and chemical states of the samples with the presence of N, O, Ti, and C. The N 1s peak of NA-165 at $400.1 \mathrm{eV}$ can be attributed to the interstitial nitrogen in the form of Ti-O-N or Ti-N-O bonds (Figure 3a) [30]. Moreover, the Ti 2p2/3 and Ti 2p1/2 core levels were located at 458.4 and $464.2 \mathrm{eV}$ (Figure 3b), which shift toward lower binding energies as compared to the reported pure $\mathrm{TiO}_{2}$ due to the nitrogen doping [31]. The nitrogen doping percentages of NA-185, NA-165, NA-145, and HA-165 were 0.66, 0.63, 0.73 , and 0.52 at.\%, respectively. Since the nitrogen content of NA-165 prepared under $\mathrm{N}_{2}$ using $\mathrm{HNO}_{3}$ as catalyst is higher than that of HA-165 prepared under $\mathrm{N}_{2}$ via the similar method under $\mathrm{N}_{2}$ but using $\mathrm{HCl}$ instead of $\mathrm{HNO}_{3}$ as catalyst, it implied that the nitrogen source in NA-165 may be from both $\mathrm{N}_{2}$ and $\mathrm{HNO}_{3}$ [22,32]. The O 1s XPS spectra of NA-165 shown in Figure 3c displays two peaks at 530.2 and $531.8 \mathrm{eV}$, which was attributed to the Ti-O bond and Ti-O-N or Ti-N-O, respectively [33]. The XPS results along with XRD patterns and HRTEM images reveal that nitrogen-doped anatase/brookite biphasic nanoparticles were successfully synthesized.

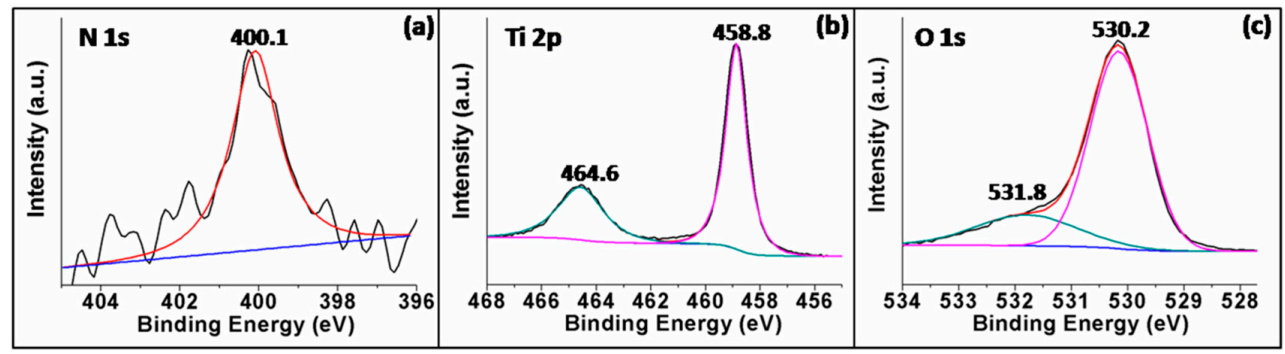

Figure 3. X-ray photoelectron spectroscopy (XPS) spectra of (a) $\mathrm{N} \mathrm{1s,} \mathrm{(b)} \mathrm{Ti} \mathrm{2p,} \mathrm{(c)} \mathrm{O} \mathrm{1s} \mathrm{region}$ for NA-165.

The nitrogen adsorption-desorption isotherms shown in Figure S3 are all Type IV, implying that the samples may have mesoporous structures. The surface areas, average pore size, and pore volumes of the samples are summarized in Table 1 and Table S1. Obviously, the surface areas of the biphasic samples changed little with the increase of heating temperature, since the surface areas of NA-145, NA-165, and NA-185 were 249, 240, and $239 \mathrm{~m}^{2} / \mathrm{g}$, respectively. HA-165 prepared using $\mathrm{HCl}$ instead of $\mathrm{HNO}_{3}$ as catalyst had lower surface area $\left(216 \mathrm{~m}^{2} / \mathrm{g}\right)$. Compared with other methods, the employed heating temperature of this work was much lower, and the heat treatment time was shorter. Nevertheless, the surface area of NA-165 was also much higher than those of many other types of nitrogen-doped anatase/brookite biphasic $\mathrm{TiO}_{2}$ except for the one treated with nitrogen plasma [22].

The UV-vis diffuse reflectance spectra of NA-185, NA-165, NA-145, and HA-165 are shown in Figure 4 using P25 as a control group. The absorbance of the N-doped samples was stronger than that 
of P25 in the visible light region. The band gap energies of NA-185, NA-165, NA-145, HA-165, and P25 were $3.05,3.03,3.01,3.09$, and $3.12 \mathrm{eV}$, respectively, which were calculated from Equation (1):

$$
\mathrm{Eg}=1240 / \lambda
$$

where Eg and $\lambda$ are the band gap energy $(\mathrm{eV})$ and wavelength of adsorption edge (nm), respectively. The narrower band gap and stronger visible-light response of the samples can be ascribed to the effect of the nitrogen doping $[3,31]$. Among the two biphasic samples, the band gap energies were increased with increasing brookite content. The reason may be due to the band gap of brookite is larger than anatase [18].

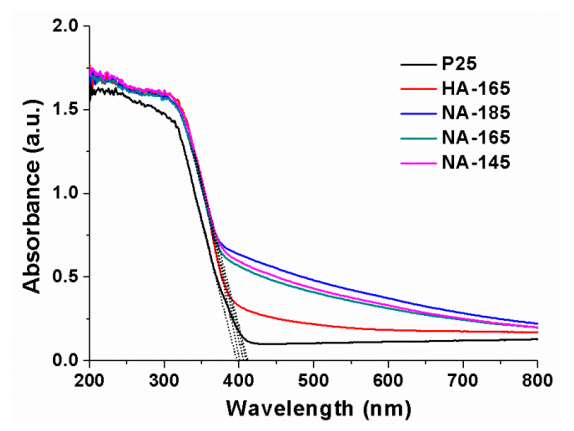

Figure 4. The UV-vis diffuse reflectance spectra of NA-145, NA-165, NA-185, HA-165, and P25.

\subsection{Photocatalytic Activity}

The visible light photocatalytic activities of as-prepared samples were tested by photodegradation of $\mathrm{MO}(10 \mathrm{mg} / \mathrm{L})$. For comparison, we have also studied the photocatalytic activities of P25 and the two samples prepared with the similar sol-gel method but heated at $165^{\circ} \mathrm{C}$ in a muffle furnace with air (MF-165) or a resistance-heated tube furnace with a flow of nitrogen (TF-165). Figure 5a shows the removal rates of MO for NA-185, NA-165, NA-145, HA-165, P25, MF-165, and TF-165 are 95\%, 92\%, $83 \%, 53 \%, 49 \%, 27 \%$, and $31 \%$, respectively. The dark reaction adsorption rates of samples are all less than $8 \%$, which implied that the removal of MO is mainly attributed to photocatalytic degradation rather than adsorption. Figure S4 shows the nitrogen-doped anatase/brookite biphasic samples of NA-185 (94\%) and NA-165 (91\%) with similar photocatalytic degradation rate of MO, which are higher than the other samples. The apparent first-order rate constant $\mathrm{K}\left(\mathrm{min}^{-1}\right)$ for NA-165 (0.021) is close to that of NA-185 (0.023), which is about 1.4, 3.5, 4.2, 9.6, and 7.5 times higher than those of NA-145, HA-165, P25, MF-165, and TF-165, respectively (Figure $5 \mathrm{~b}$ and Table 1). Thus, $165^{\circ} \mathrm{C}$ was chosen as a reasonable heating treatment temperature.

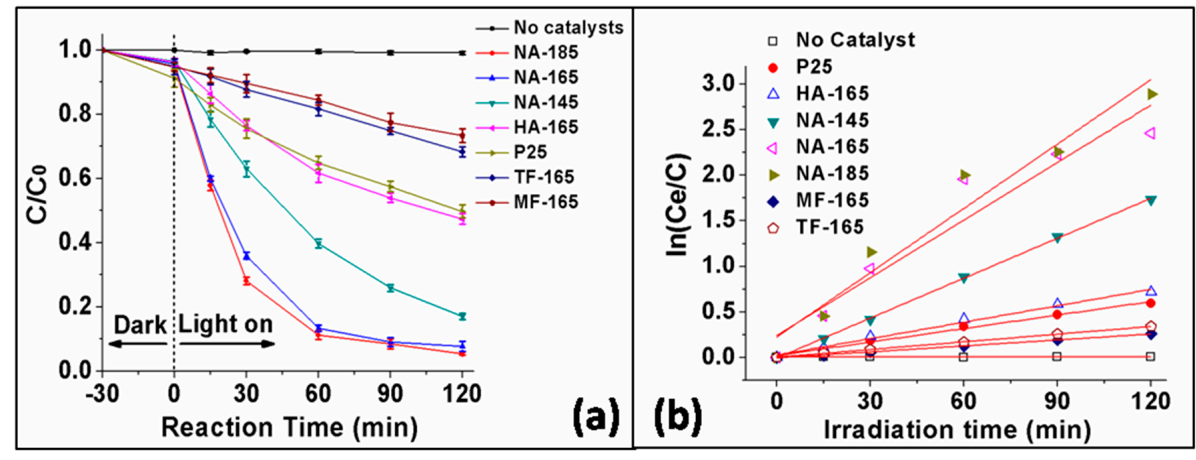

Figure 5. (a) Removal curves of methyl orange (MO). Error bars represent the standard deviation from three measurements; (b) Apparent first-order kinetics plot for the photocatalytic degradation of $\mathrm{MO}$ over different samples. 
Table 2 summarizes the preparation methods, surface area, and visible-light photocatalytic activity of nitrogen-doped anatase/brookite biphasic $\mathrm{TiO}_{2}$ reported in recent years. The 4.2 -fold visible light activity enhancement as compared to P25 suggests that NA-165 is a potential highly efficient photocatalyst. By contrast, if the aged gel was heated in amuffle furnace with air or obtained in a resistance-heated tube furnace with a flow of nitrogen at the same temperature $\left(165^{\circ} \mathrm{C}\right)$, respectively, only amorphous samples were obtained in spite of large surface area (Figure S5 and Table 1). Meanwhile, they were much less active compared to NA-165. This implies that the crystallinity may play a more important role. Moreover, the presence of brookite in the mixture can reduce the recombination of hole-electron pairs. The band gap was also widened with increasing brookite content [34]. This is why NA-165 (3.03 eV, 6\% brookite content) and NA-185 (3.05 eV, 10\% brookite content) exhibited similar MO photocatalytic degradation activity.

The photocatalytic stability of NA-165 was tested by cycling experiments. For each cycling run, NA-165 was separated by centrifugation, and dried at $90^{\circ} \mathrm{C}$. As shown in Figure 6, there was no significant decrease of photocatalytic degradation rate after three cycling runs. This result suggested that NA-165 was a stable photocatalyst for organic dye degradation under visible light.

Table 2. Comparison of nitrogen-doped anatase/brookite biphasic $\mathrm{TiO}_{2}$ prepared by various methods.

\begin{tabular}{|c|c|c|c|c|c|}
\hline Sample & $\begin{array}{c}\text { Surface } \\
\text { Area }\left(\mathrm{m}^{2} / \mathrm{g}\right)\end{array}$ & Preparation Method & Application & $\begin{array}{l}\text { The Times of } K \\
\left(\mathrm{~min}^{-1}\right) \text { to } P 25\end{array}$ & Reference \\
\hline $\begin{array}{l}\text { Nitrogen-doped } \mathrm{TiO}_{2} \text { nanorods } \\
\text { with anatase/brookite structures }\end{array}$ & 51.1 & $\begin{array}{c}\text { Hydrothermal } \\
\text { synthesis } 200^{\circ} \mathrm{C}, 48 \mathrm{~h}\end{array}$ & $\begin{array}{c}\text { Degradation of MO } \\
\text { and 4-chlorophenol } \\
(4-\mathrm{CP})\end{array}$ & $2.3,2.7$ & [19] \\
\hline $\begin{array}{c}\text { Nitrogen-doped anatase/brookite } \\
\text { titania }\end{array}$ & 124.4 & $\begin{array}{l}\text { Solvothermal } \\
\text { synthesis } 190^{\circ} \mathrm{C}, 3 \mathrm{~h}\end{array}$ & Degradation of MO & & [24] \\
\hline Nitrogen-doped $\mathrm{TiO}_{2}$ film & & $\begin{array}{c}\text { Solvothermal } \\
\text { synthesis } 180^{\circ} \mathrm{C}, 18 \mathrm{~h}\end{array}$ & Degradation of MB & & {$[35]$} \\
\hline $\begin{array}{c}\text { Bicrystalline (anatase/brookite) } \\
\text { nanoporous nitrogen-doped } \mathrm{TiO}_{2}\end{array}$ & 375.9 & $\begin{array}{l}\text { Plasma treatment } \\
0.5 \mathrm{~h} \\
\end{array}$ & $\begin{array}{c}\text { Degradation of } \\
\text { Rhodamine B (RhB) }\end{array}$ & & [23] \\
\hline
\end{tabular}

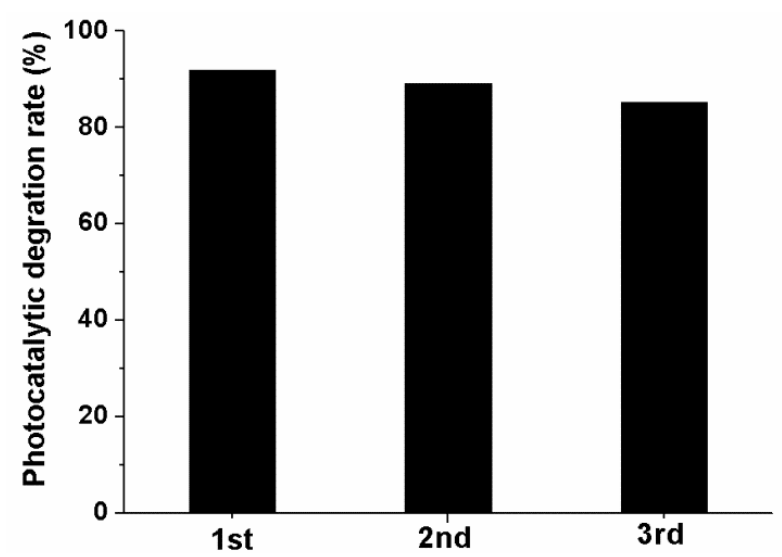

Figure 6. Cycling run in the photocatalytic degradation of MO under visible light over NA-165.

\subsection{Possible Reasons for the Enhancement of the Visible-Light Performance}

It is interesting to evoke some reasons why NA-165 has high visible-light performance, though mechanism of the enhancement is still far from understood. The first explanation is that the absorption edge of NA-165 shifts to the visible-light range, and then they possess narrower band gap, and have definite absorptions in the visible region due to the presence of nitrogen-doping, which has been confirmed by UV-vis diffuse reflectance spectra and XPS study. Secondly, the anatase/brookite biphasic 
nanoparticles are aggregated closely, as shown in Figures S1 and S2. The intimate contact can facilitate inter particle charge transfer from brookite to anatase and reduce the recombination of electron-hole pairs. Thirdly, the large surface area can provide more active sites and improve the diffusion and migration of $\mathrm{MO}$ in the process of photodegradation [36]. Furthermore, the competitive diffusion of the $\mathrm{H}_{2} \mathrm{O}$ and dye molecules, dye molecule structure, and photocatalytic degradation route are also the factors influencingthe photocatalytic process [37]. The photodegradation of $\mathrm{MO}$ under visible light was mainly driven by the active species $\mathrm{O}_{2}^{\bullet-}, \mathrm{h}^{+}$, and $\bullet$ OH [38].

\section{Materials and Methods}

\subsection{Synthesis}

Titanium tetraisopropoxide (TTIP, $\geq 97 \%$, Sigma-Aldrich, St. Louis, MO, USA) was of chemical grade. Acetone ( $\geq 99.5 \%$, Tianjin Fengchuan Chemical Reagent Technologies Co., Ltd., Tianjin, China), $\mathrm{HNO}_{3}(65 \%$, Xilong Scientific Co., Ltd., Shantou, China), and acetylacetone ( $\geq 98 \%$, Tianjin Fengchuan Chemical Reagent Technologies Co., Ltd., Tianjin, China) were of analytical grade. All chemicals were used without further purification.

Nitrogen-doped anatase/brookite biphasic nanoparticles were prepared with a sol-gel process modified from a sol-gel combined solvothermal route [24]. Titanium tetraisopropoxide, acetone, $\mathrm{HNO}_{3}$ and acetylacetone with the volume ratio of 6.5:20:0.11:0.54 were mixed in a glass beaker. A mixture solution of deionized water and acetone (volume ratio of 1.2:7.5) was then added dropwise with vigorous stirring until reaching the gelling point. The gels were placed into a quartz-lined stainless-steel autoclave after being aged for $24 \mathrm{~h}$ at room temperature. Then, the temperature of the autoclave was increased to and held at 145,165 , or $185^{\circ} \mathrm{C}$ for $4 \mathrm{~h}$ under nitrogen atmosphere after flushing the autoclave with nitrogen gas. The initial and final pressures were under atmospheric and autogenous pressure, respectively. After the heat treatment ended, the pressure was released quickly to remove the solvent vapour. The obtained material was cooled down to room temperature by nitrogen purging before being washed with deionized water and dried in vacuum at $90^{\circ} \mathrm{C}$ for $4 \mathrm{~h}$. According to the heat treatment temperature, the samples were denoted as NA-145, NA-165, and NA-185, respectively. If $\mathrm{HNO}_{3}$ was replaced by the same volume of $\mathrm{HCl}$ in the process of preparation, then the sample was denoted as HA-165.

\subsection{Photocatalytic Activity}

In each experiment, $50 \mathrm{mg}$ photocatalysts and $50 \mathrm{~mL}$ of $\mathrm{MO}$ solution $(10 \mathrm{mg} / \mathrm{L})$ was placed in a glass vessel with a cooling water jacket and quartz cover. The suspensions were stirred in the dark for $30 \mathrm{~min}$ to reach the adsorption-desorption equilibrium. Then, the system was exposed for $120 \mathrm{~min}$ under visible light irradiation provided by a $500 \mathrm{~W}$ Xe lamp with a $420 \mathrm{~nm}$ cut off filter. At certain time intervals, $3 \mathrm{~mL}$ of the suspensions was collected and centrifuged (10,000 rpm, $20 \mathrm{~min})$ to remove the photocatalysts. The separated solution was analysed and the maximum absorption was recorded at $464 \mathrm{~nm}$ by a spectrophotometer (Shimadzu UV-2600, Kyoto, Japan).

The removal rate of $\mathrm{MO}$ was calculated using Equation (2):

$$
\text { removal rate }=\mathrm{C} / \mathrm{C}_{0}
$$

where $C$ and $C_{0}$ are the initial and instantaneous absorbance of $\mathrm{MO}$ at $464 \mathrm{~nm}$.

The photocatalytic degradation rates of $\mathrm{MO}$ and the first-order rate constant $\mathrm{K}\left(\mathrm{min}^{-1}\right)$ were calculated, respectively, using the Equations (3) and (4):

$$
\begin{gathered}
\text { photocatalytic degradation rate }=\mathrm{C} / \mathrm{Ce}, \\
\ln (\mathrm{Ce} / \mathrm{C})=\mathrm{Kt},
\end{gathered}
$$


where $\mathrm{Ce}$ and $\mathrm{C}$ are the adsorption-desorption equilibrium absorbance and instantaneous absorbance of $\mathrm{MO}$ at $464 \mathrm{~nm}$, respectively. $\mathrm{t}$ is the irradiation time.

\subsection{Characterizations}

X-ray powder diffraction (XRD, Rigaku Co., Tokyo, Japan) analysis was conducted on a D/max-3B spectrometer with $\mathrm{Cu} \mathrm{K} \alpha$ radiation at a range from $10^{\circ}$ to $90^{\circ}(2 \theta)$. Brunauer-Emmett-Teller (BET) surface area, pore volume, and pore size were measured by nitrogen adsorption/desorption using a Micromeritics Tristar II Surface Area and Porosity Analyzer (Micromeritics, Norcross, GA, USA). Transmission electron microscopy (TEM) was conducted on a Hitachi H-800 instrument (Japan Electron Optics Laboratory Co., Ltd., Tokyo, Japan). Scanning electron microscopy (SEM) images were taken on a FEIQuanta200FEG microscope (FEI, Hillsboro, OR, USA). X-ray photoelectron spectroscopy (XPS) was recorded using a Thermo Fisher Scientific K-Alpha ${ }^{+}$XPS system with Al K $\alpha$ radiation and adventitious C1s peak ( $284.8 \mathrm{eV}$ ) calibration (Thermo Fisher Scientific Inc., Waltham, MA, USA). UV-Vis diffuse reflectance spectra were measured on a UV-2600 photometer (Shimadzu Corp., Kyoto, Japan).

\section{Conclusions}

The nitrogen-doped anatase/brookite biphasic nanoparticles with large surface area $\left(240 \mathrm{~m}^{2} / \mathrm{g}\right)$ were successfully prepared during the sol-gel preparation at low temperature $\left(165{ }^{\circ} \mathrm{C}, 4 \mathrm{~h}\right)$. The sample obtained without subsequent annealing at elevated temperature, which exhibited enhanced visible-light photocatalytic efficiency for the degradation of MO with 4.2-, 9.6-, and 7.5-fold visible light activities as compared to P25, MF-165, and TF-165, respectively. This was attributed to nitrogen doping, mixed crystalline phase, and high surface area. The recycling experiments suggested that NA-165 was a stable visible-light photocatalyst. The sample and low-temperature synthetic method developed in this work may provide a new pathway to prepare the stable photocatalyst for the degradation of organic dyes under visible light.

Supplementary Materials: The following are available online at www.mdpi.com/2073-4344/7/12/376/s1, Figure S1: Scanning electron microscopy (SEM) images of NA-185 (a), NA-165 (b), NA-145 (c) and HA-145 (d); Figure S2: Transmission electron microscopy (TEM) Transmission electron microscopy (TEM); Figure S3: Nitrogen adsorption-desorption isotherms for the prepared samples; Figure S4: Photocatalytic degradation of MO under visible light over different samples; Figure S5: XRD patterns of MF-165 and TF-165; Table S1: The Brunauer-Emmett-Teller (BET)analysis data of samples.

Acknowledgments: The work was supported by National Natural Science Foundation of China (Project 21403190, 21573193, 21367024, 21464016 and 21263027). The authors also thank Program for Innovation Team of Yunnan Province and Innovative Research Team (in Science and Technology) in the Universities of Yunnan Province, Key Laboratory of Advanced Materials for Wastewater Treatment of Kunming, the Key project from the Yunnan Educational Committee (Project ZD2012003), Yunnan Provincial Natural Science Foundation (Project 2015FB106) and Yunnan Applied Basic Research Projects (Project 2016FA002) for financial support.

Author Contributions: Jiaqiang Wang and Liang Jiang conceived and designed the experiments; Liang Jiang, Yizhou Li, and Haiyan Yang performed the experiments; Yepeng Yang, Xiang Long and Jun Liu analyzed the data; Liang Jiang, Zhiying Yan, and Jiao He wrote the paper; Jiaqiang Wang and Zhiying Yan modified the paper.

Conflicts of Interest: The authors declare no conflict of interest.

\section{References}

1. Zhang, W.; Zou, L.; Wang, L. Photocatalytic $\mathrm{TiO}_{2}$ /adsorbent nanocomposites prepared via wet chemical impregnation for wastewater treatment: A review. Appl. Catal. A 2009, 371, 1-9. [CrossRef]

2. Zhang, Z.; Wang, X.; Long, J.; Gu, Q.; Ding, Z.; Fu, X. Nitrogen-doped titanium dioxide visible light photocatalyst: Spectroscopic identification of photoactive centers. J. Catal. 2010, 276, 201-214. [CrossRef]

3. Ryu, J.; Choi, W. Substrate-specific photocatalytic activities of $\mathrm{TiO}_{2}$ and multiactivity test for water treatment application. Environ. Sci. Technol. 2008, 42, 294-300. [CrossRef] [PubMed]

4. Asahi, R.; Morikawa, T.; Ohwaki, T.; Aoki, K.; Taga, Y. Visible-light photocatalysis in nitrogen-doped titanium oxides. Science 2001, 293, 269-271. [CrossRef] [PubMed] 
5. Valentin, C.D.; Finazzi, E.; Pacchioni, G.; Selloni, A.; Livraghi, S.; Paganini, M.C.; Giamello, E. N-doped TiO 2 : Theory and experiment. Chem. Phys. 2007, 339, 44-56. [CrossRef]

6. Wang, H.; Gao, X.; Duan, G.; Yang, X.; Liu, X. Facile preparation of anatase-brookite-rutile mixed-phase $\mathrm{N}$-doped $\mathrm{TiO}_{2}$ with high visible-light photocatalytic activity. J. Environ. Chem. Eng. 2015, 3, $603-608$. [CrossRef]

7. Li, H.; Li, J.; Huo, Y. Highly Active $\mathrm{TiO}_{2} \mathrm{~N}$ Photocatalysts Prepared by Treating $\mathrm{TiO}_{2}$ Precursors in $\mathrm{NH}_{3}$ /Ethanol Fluid under Supercritical Conditions. J. Phys. Chem. B 2006, 110, 1559-1565. [CrossRef] [PubMed]

8. Paola, A.D.; Bellardita, M.; Palmisano, L. Brookite, the Least Known $\mathrm{TiO}_{2}$ Photocatalyst. Catalysts 2013, 3 , 36-73. [CrossRef]

9. Reyes-Coronado, D.; Rodríguez-Gattorno, G.; Espinosa-Pesqueira, M.E.; Cab, C.; de Coss, R.; Oskam, G. Phase-pure $\mathrm{TiO}_{2}$ nanoparticles: Anatase, brookite and rutile. Nanotechnology 2008, 19, 145605. [CrossRef] [PubMed]

10. Yu, J.; Yu, J.C.; Leung, M.K.P.; Ho, W.; Cheng, B.; Zhao, X.; Zhao, J. Effects of acidic and basic hydrolysis catalysts on the photocatalytic activity and microstructures of bimodal mesoporous titania. J. Catal. 2003, 217, 69-78. [CrossRef]

11. Zhao, B.; Chen, F.; Jiao, Y.; Yang, H.; Zhang, J. Ag ${ }^{0}$-loaded brookite/anatase composite with enhanced photocatalytic performance towards the degradation of methyl orange. J. Mol. Catal. A Chem. 2011, 348, 114-119. [CrossRef]

12. Ozawa, T.; Iwasaki, M.; Tada, H.; Akita, T.; Tanaka, K.; Ito, S. Low-temperature synthesis of anatase-brookite composite nanocrystals: The junction effect on photocatalytic activity. J. Colloid Interface Sci. 2005, 281, 510-513. [CrossRef] [PubMed]

13. Kandiel, T.A.; Feldhoff, A.; Robben, L.; Dillert, R.; Bahnemann, D.W. Tailored Titanium Dioxide Nanomaterials: Anatase Nanoparticles and Brookite Nanorods as Highly Active Photocatalysts. Chem. Mater. 2010, 22, 2050-2060. [CrossRef]

14. Mutuma, B.K.; Shao, G.N.; Kim, W.D.; Kim, H.T. Sol-gel synthesis of mesoporous anatase-brookite and anatase-brookite-rutile $\mathrm{TiO}_{2}$ nanoparticles and their photocatalytic properties. J. Colloid Interface Sci. 2015, 442, 1-7. [CrossRef] [PubMed]

15. Scanlon, D.O.; Dunnill, C.W.; Buckeridge, J.; Shevlin, S.A.; Logsdail, A.J.; Woodley, S.M.; Catlow, C.R.A.; Powell, M.J.; Palgrave, R.G.; Parkin, I.P.; et al. Band alignment of rutile and anatase $\mathrm{TiO}_{2}$. Nat. Mater. 2013, 12, 798-801. [CrossRef] [PubMed]

16. Mohamed, M.A.; Salleh, W.N.W.; Jaafar, J.; Ismail, A.F.; Nor, N.A.M. Photodegradation of phenol by $\mathrm{N}$-Doped $\mathrm{TiO}_{2}$ anatase/rutile nanorods assembled microsphere under UV and visible light irradiation. Mater. Chem. Phys. 2015, 162, 113-123. [CrossRef]

17. Nie, X.; Zhuo, S.; Maeng, G.; Sohlberg, K. Doping of $\mathrm{TiO}_{2}$ Polymorphs for Altered Optical and Photocatalytic Properties. Int. J. Photoenergy 2009, 2009, 294042. [CrossRef]

18. Yu, J.C.; Yu, J.; Ho, W.; Zhang, L. Preparation of highly photocatalytic active nano-sized $\mathrm{TiO}_{2}$ particles via ultrasonic irradiation. Chem. Commun. 2001, 19, 1942-1943. [CrossRef]

19. Gai, L.; Duan, X.; Jiang, H.; Mei, Q.; Zhou, G.; Tian, Y.; Liu, H. One-pot synthesis of nitrogen-doped $\mathrm{TiO}_{2}$ nanorods with anatase/brookite structures and enhanced photocatalytic activity. CrystEngComm 2012, 14, 7662-7671. [CrossRef]

20. El-Sheikh, S.M.; Khedr, T.M.; Zhang, G.; Vogiazi, V.; Ismail, A.A.; O'Shea, K.; Dionysiou, D.D. Tailored synthesis of anatase-brookite heterojunction photocatalysts for degradation of cylindrospermopsin under UV-Vis light. Chem. Eng. J. 2017, 310, 428-436. [CrossRef]

21. Etacheri, V.; Seery, M.K.; Hinder, S.J.; Pillai, S.C. Highly Visible Light Active $\mathrm{TiO}_{2-x} \mathrm{~N}_{x}$ Heterojunction Photocatalysts. Chem. Mater. 2010, 22, 3843-3853. [CrossRef]

22. Li, L.; Liu, C. Facile Synthesis of Anatase-Brookite Mixed-Phase N-Doped $\mathrm{TiO}_{2}$ Nanoparticles with High Visible-Light Photocatalytic Activity. Eur. J. Inorg. Chem. 2009, 25, 3727-3733. [CrossRef]

23. Lee, H.U.; Lee, Y.; Lee, S.C.; Park, S.Y.; Son, B.; Lee, J.W.; Lim, C.; Choi, C.; Choi, M.; Lee, S.Y.; et al. Visible-light-responsive bicrystalline (anatase/brookite) nanoporous nitrogen-doped $\mathrm{TiO}_{2}$ photocatalysts by plasma treatment. Chem. Eng. J. 2014, 254, 268-275. [CrossRef]

24. Liu, J.; Qin, W.; Zuo, S.; Yu, Y.; Hao, Z. Solvothermal-induced phase transition and visible photocatalytic activity of nitrogen-doped titania. J. Hazard. Mater. 2009, 163, 273-278. [CrossRef] [PubMed] 
25. Lin, L.; Huang, J.; Li, X.; Abass, M.A.; Zhang, S. Effective surface disorder engineering of metal oxide nanocrystals forimproved photocatalysis. Appl. Catal. B 2017, 203, 615-624. [CrossRef]

26. Zimbone, M.; Cacciato, G.; Boutinguiza, M.; Privitera, V.; Grimaldi, M.G. Laser irradiation in water for the novel, scalable synthesis of black $\mathrm{TiO}_{x}$ photocatalyst for environmental remediation. Beilstein J. Nanotechnol. 2017, 8, 196-202. [CrossRef] [PubMed]

27. Zhao, Z.; Jiao, X.; Chen, D. Preparation of $\mathrm{TiO}_{2}$ aerogels by a sol-gel combined solvothermal route. J. Mater. Chem. 2009, 19, 3078-3083. [CrossRef]

28. Wang, J.; Uma, S.; Klabunde, K.J. Visible light photocatalysis in transition metal incorporated titania-silica aerogels. Appl. Catal. B 2004, 48, 151-154. [CrossRef]

29. Li, J.; Ishigaki, T.; Sun, X. Anatase, Brookite, and Rutile Nanocrystals via Redox Reactions under Mild Hydrothermal Conditions: Phase-Selective Synthesis and Physicochemical Properties. J. Phys. Chem. C 2007, 111, 4969-4976. [CrossRef]

30. Jagadale, T.C.; Takale, S.P.; Sonawane, R.S.; Joshi, H.M.; Patil, S.I.; Kale, B.B.; Ogale, S.B. N-Doped TiO 2 Nanoparticle Based Visible Light Photocatalyst by Modified Peroxide Sol-Gel Method. J. Phys. Chem. C 2008, 112, 14595-14602. [CrossRef]

31. Li, J.; Xu, X.; Liub, X.; Qin, W.; Pan, L. Novel cake-like N-doped anatase/rutile mixed phase $\mathrm{TiO}_{2}$ derived from metal-organic frameworks for visible light photocatalysis. Ceram. Int. 2017, 43, 835-840. [CrossRef]

32. Zhang, Y.C.; Yang, M.; Zhang, G.; Dionysiou, D.D. $\mathrm{HNO}_{3}$-involved one-step low temperature solvothermal synthesis of $\mathrm{N}$-doped $\mathrm{TiO}_{2}$ nanocrystals for efficient photocatalytic reduction of $\mathrm{Cr}(\mathrm{VI})$ in water. Appl. Catal. B 2013, 142, 249-258. [CrossRef]

33. György, E.; del Pino, A.P.; Serra, P.; Morenza, J.L. Depth profiling characterisation of the surface layer obtained by pulsed Nd:YAG laser irradiation of titanium in nitrogen. Surf. Coat. Technol. 2003, 173, 265-270. [CrossRef]

34. Zhao, H.; Liu, L.; Andinobc, J.M.; Li, Y. Bicrystalline $\mathrm{TiO}_{2}$ with controllable anatase-brookite phase content for enhanced $\mathrm{CO}_{2}$ photoreduction to fuels. J. Mater. Chem. A 2013, 1, 8209-8216. [CrossRef]

35. Wang, J.; Mao, B.; Gole, J.L.; Burda, C. Visible-light-driven reversible and switchable hydrophobic to hydrophilic nitrogen-doped titania surfaces: Correlation with photocatalysis. Nanoscale 2010, 2, 2257-2261. [CrossRef] [PubMed]

36. Yan, Z.; He, J.; Guo, L.; Li, Y.; Duan, D.; Chen, Y.; Li, J.; Yuan, F.; Wang, J. Biotemplated Mesoporous $\mathrm{TiO}_{2} / \mathrm{SiO}_{2}$ Composite Derived from Aquatic Plant Leaves for Efficient Dye Degradation. Catalysts 2017, 7, 82. [CrossRef]

37. Miao, Y.; Zhai, Z.; Jiang, L.; Shi, Y.; Yan, Z.; Duan, D.; Zhen, K.; Wang, J. Facile and new synthesis of cobalt doped mesoporous $\mathrm{TiO}_{2}$ with high visible-light performance. Powder Technol. 2014, 266, 365-371. [CrossRef]

38. Li, W.; Li, D.; Lin, Y.; Wang, P.; Chen, W.; Fu, X.; Shao, Y. Evidence for the Active Species Involved in the Photodegradation Process of Methyl Orange on $\mathrm{TiO}_{2}$. J. Phys. Chem. C 2012, 116, 3552-3560. [CrossRef] 